\title{
Feedback Control Strategies \\ for a Nonholonomic Mobile \\ Robot Using a \\ Nonlinear Oscillator
}

\section{Ranjan Mukherjee}

Department of Mechanical Engineering

Michigan State University

East Lansing, Michigan 48824

e-mail:mukherji@egr.msu.edu

\section{Degang Chen}

Department of Electrical and

Computer Engineering

Iowa State University

Ames, Iowa 50011

\section{Gangbing Song}

Department of Mechanical Engineering

University of Akron

Akron, Ohio 44325

Received June 4, 1998; accepted November 14, 1998

\begin{abstract}
Among control problems for mobile robots, point-to-point stabilization is the most challenging since it does not admit designs with smooth static state feedback laws. Stabilization strategies for mobile robots, and nonholonomic systems generally, are smooth, time-varying or nonsmooth, time-invariant. Time-varying control strategies are designed with umdamped linear oscillators but their fixed structure offer limited flexibility in control design. The central theme of this paper lies in use of nonlinear oscillators for mobile robot control. Large numbers of qualitatively different control strategies can be designed using nonlinear oscillators since stiffness and damping can be functions of robot states. We demonstrate by designing two fundamentally different controllers for two-wheeled mobile robot using two variants of a particular nonlinear oscillator. First controller is dynamic and generates smooth control action.
\end{abstract}

* To whom all correspondence should be addressed. 
Second controller is almost-smooth and time-invariant. While first controller guarantees global asymptotic stability for any desired posture of robot, second controller is stable, and converges robot from almost any posture to desired posture. The only gap in posture space is unstable equilibrium manifold of measure zero. For both control strategies we mathematically establish stability and convergence of mobile robot to desired posture. Simulation results support theoretical claims. (c) 1999 John Wiley \& Sons, Inc.

\section{INTRODUCTION}

As a bench-mark problem for nonholonomic systems, mobile robot control has been quite popular in the scientific community. Among the control problems, the problem of point-to-point stabilization is particularly interesting due to the nonexistence of a smooth stabilizing static state feedback law. This negative result is a consequence of a theorem by Brockett. ${ }^{1}$ In agreement with Brockett's ${ }^{1}$ result, primarily two types of stabilizing controllers have been developed for nonholonomic systems, namely, smooth time-varying controllers and nonsmooth time-invariant controllers. A hybrid combination of the two controllers have also been proposed. A summary of the developments in mobile robot control, and nonholonomic systems in general, can be found in review articles. ${ }^{2}$

Explicit time-varying control laws for the feedback stabilization of a mobile robot was first proposed by Samson. ${ }^{3}$ Several studies have been carried out thereafter on smooth time-varying control of nonholonomic systems. These include general existence results ${ }^{4}$ and explicit time-periodic feedback laws for a particular class of nonlinear systems. ${ }^{5}$ Dynamic controllers, which are a special case of smooth time-varying controllers, have also been proposed. 6 Smooth time-varying controllers typically suffer from slow rates of convergence ${ }^{7,8}$ and faster convergence can be achieved through the design of nonsmooth controllers. The existence of a piecewise smooth stabilizing controller for nonholonomic systems was shown by Sussman ${ }^{9}$ but the work on such control methods was initiated by Bloch, McClamroch, and Reyhanoglu. ${ }^{10}$ Piecewise smooth controllers with exponential convergence were subsequently developed for mobile robots. ${ }^{11}$ In a different approach to constructing piecewise smooth controllers, ${ }^{12,13}$ a nonsmooth state transformation was used and a smooth time-invariant controller was designed in the transformed coordinates. The feedback law in the original coordinates is, however, discontinuous. Discontinuous controllers based on sliding modes have also been proposed, ${ }^{14,15}$ but such controllers sometimes lead to chattering.
Hybrid controllers, proposed for nonholonomic systems, are based on switchings at discrete-time instants between various low level continuous-time controllers. Such controllers have been proposed by a few authors. . $^{16,17}$

In this article we introduce a new method for the design of feedback control strategies for mobile robots. The central theme lies in the use of nonlinear oscillators that offer flexibility in control design. Undamped linear oscillators ${ }^{5}$ have been widely used in time-varying control of mobile robots and nonholonomic systems but they offer limited flexibility due to their fixed structure. A large number of variations are possible using nonlinear oscillators since their stiffness and damping can be functions of the mobile robot states. In this article we use two nonlinear oscillators with the same structure to design two fundamentally different control strategies for the two-wheeled mobile robot. The oscillator frequency is constant in the first controller while it is a function of the mobile robot states in the second design. As different from control designs with linear oscillators, the oscillator states in both our controllers converge to the origin along with the states of the mobile robot. This is a result of physically motivated control designs where the oscillator states are chosen to be the states of the mobile robot.

The first controller presented in this article is a dynamic controller. It generates smooth control action and guarantees global asymptotic stability for any desired posture of the mobile robot. The controller uses the dynamics of a nonlinear oscillator to prevent the robot from getting stuck at any point other than the desired posture, and in this regard it is functionally similar to existing time-periodic controllers using linear oscillators. The nonlinear damping of the oscillator, however, makes our controller qualitatively different from existing time-periodic controllers. The second control design is quite different from existing control strategies for mobile robots and nonholonomic systems. It generates almost-smooth ${ }^{18}$ time-invariant control action that can asymptotically converge the robot from practically any posture to the desired posture. The only excep- 
tion is an equilibrium manifold of measure zero (containing the desired posture) from where the robot cannot move. All points on this manifold (except the desired posture) however, are rendered unstable and therefore any posture arbitrarily close to, but not exactly on, the manifold can be asymptotically converged to the desired posture. The controller guarantees stability of the desired posture and is simple in structure and easy to implement. Both controllers can handle situations where the robot has to turn more than $360^{\circ}$ between initial and final configurations. Such maneuvers are needed in factor automation to unwind tethers such as the paint supply-line during spray painting or the current cable during welding. In such situations, typically, the exact point of the turn is not important.

Although both controllers use structurally similar nonlinear oscillators, the resulting closed loop systems are quite different. The first controller is dynamic and the control action is smooth. In contrast, the control action of the second controller is almost-smooth and time-invariant. The first controller leads to global asymptotic stability of the desired posture, while the second leaves a gap of measure zero in the posture space from where the robot cannot be converged to the desired posture. The first controller uses the kinematics of the mobile robot in standard form while the second controller provides a means of generalization by using the chained form. ${ }^{19}$ These differences demonstrate the strengths and flexibility of the nonlinear oscillators used. In the past nonlinear oscillators have not been used for the control of nonholonomic systems and in this article we show that they can be used in a creative way for the control of mobile robots.

This article is organized as follows. Two kinematic models of the mobile robot are presented in section 2. In section 3 we present the nonlinear oscillators used for controller designs. The two controllers are presented in sections 4 and 5. In these sections we also establish the stability and convergence of the closed loop system trajectories. Simulation results are presented in section 6 and section 7 contains concluding remarks.

\section{KINEMATICS OF A TWO-WHEELED MOBILE ROBOT}

The kinematics of a two-wheeled mobile robot, shown in Figure 1, is given as

$$
\dot{x}=\cos \theta u_{1}
$$

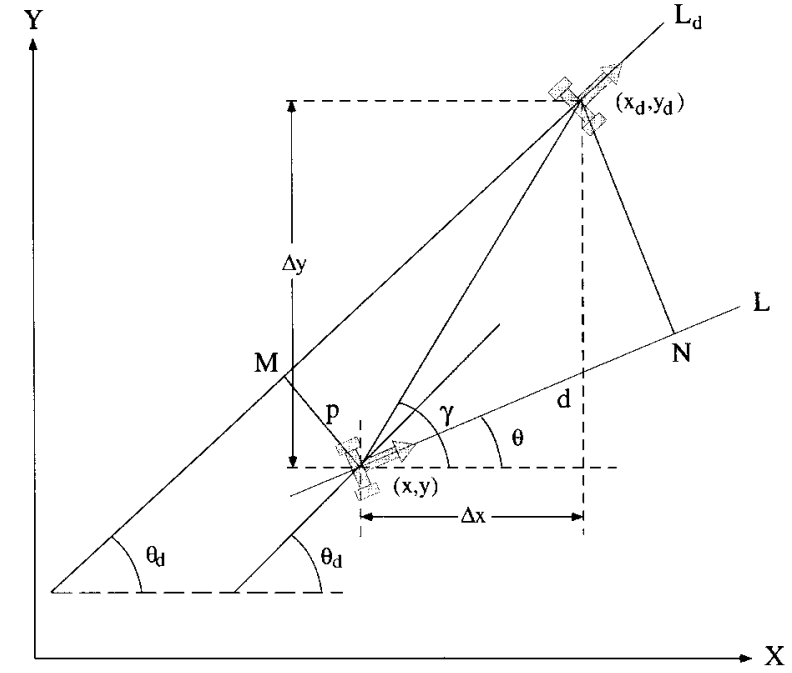

Figure 1. Diagram showing the initial and final configurations of the mobile robot and defining the variables $p$ and $d$ with respect to the initial and final configurations.

$$
\begin{aligned}
& \dot{y}=\sin \theta u_{1} \\
& \dot{\theta}=u_{2}
\end{aligned}
$$

where, $x$ and $y$ denote the Cartesian coordinates of the robot and $\theta$ denotes the orientation of the robot with respect to the positive $x$ axis. $u_{1}$ and $u_{2}$ are the linear and angular velocities of the robot, they are also the controls inputs.

In reference to Figure 1 , it is assumed that $x, y$, and $\theta$ denote the current configuration of the robot, and $x_{d}, y_{d}$, and $\theta_{d}$ denote the desired configuration. Let $L$ represent the straight line passing through the coordinates $x$ and $y$ with the slope of $\theta$, and let $L_{d}$ represent the straight line passing through the coordinates $x_{d}$ and $y_{d}$ with the slope of $\theta_{d}$. Let $M$ and $N$ denote the orthogonal projection of the point $(x, y)$ on $L_{d}$ and $\left(x_{d}, y_{d}\right)$ on $L$, respectively. If $p$ denotes the distance between the points $M$ and $(x, y)$, and $d$ denotes the distance between points $N$ and $(x, y)$, they can be expressed as follows,

$$
\begin{aligned}
p & =\left(\Delta x^{2}+\Delta y^{2}\right)^{1 / 2} \sin \left(\gamma-\theta_{d}\right) \\
& =\Delta y \cos \theta_{d}-\Delta x \sin \theta_{d} \\
d & =\left(\Delta x^{2}+\Delta y^{2}\right)^{1 / 2} \cos (\gamma-\theta) \\
& =\Delta x \cos \theta+\Delta y \sin \theta
\end{aligned}
$$

where $\Delta x$ and $\Delta y$ are defined as $\Delta x \triangleq\left(x_{d}-x\right)$ and $\Delta y \triangleq\left(y_{d}-y\right)$, respectively. If we additionally define $\Delta \theta \triangleq\left(\theta_{d}-\theta\right)$, then the kinematics of the robot 
is transformed to the form,

$$
\begin{aligned}
\dot{p} & =u_{1} \sin (\Delta \theta) \\
\dot{d} & =-u_{1} \\
\Delta \dot{\theta} & =-u_{2}
\end{aligned}
$$

The task of converging the mobile robot from its present coordinates $(x, y, \theta)$ to the desired coordinates $\left(x_{d}, y_{d}, \theta_{d}\right)$ is equivalent to the task of converging the new variables $(p, d, \Delta \theta)$ to $(0,0,0)$.

To obtain a second kinematic model, we begin with the assumption that $x_{d}, y_{d}$, and $\theta_{d}$ are all zero. If they are not zero, we can use a translation and rotation of the coordinates to set them equal to zero. Now consider the following state and control transformation $^{3}$ of the kinematic equations given by Eq. (1),

$$
\begin{aligned}
& x_{1}=\theta \\
& x_{2}=x \cos \theta+y \sin \theta \\
& x_{3}=x \sin \theta-y \cos \theta \\
& v_{1}=u_{2} \\
& v_{2}=u_{1}-(x \sin \theta-y \cos \theta) u_{2}
\end{aligned}
$$

Then, it can be easily verified that the transformed kinematics takes the chained form,

$$
\begin{aligned}
& \dot{x}_{1}=v_{1} \\
& \dot{x}_{2}=v_{2} \\
& \dot{x}_{3}=x_{2} v_{1}
\end{aligned}
$$

The task of converging the mobile robot from its present coordinates to the desired coordinates is now equivalent to the task of converging the variables $\left(x_{1}, x_{2}, x_{3}\right)$ to $(0,0,0)$.

\section{A NONLINEAR OSCILLATOR FOR MOBILE ROBOT CONTROL}

In the past smooth time-varying controllers have been designed for mobile robots and other nonholonomic systems. These controllers use the undamped linear oscillator,

$$
\begin{aligned}
& \dot{z}_{1}=-\omega z_{2} \\
& \dot{z}_{2}=\omega z_{1}
\end{aligned}
$$

The state of the oscillator is a sinusoidal function of time and time-varying controllers use feedback based on the states of the robot and the oscillator.

For the two-wheeled mobile robot, we use the following nonlinear oscillator, ${ }^{20}$

$$
\begin{aligned}
& \dot{z}_{1}=-\mu z_{1}\left[z_{1}^{2}+z_{2}^{2}-n W\right]-\omega z_{2} \\
& \dot{z}_{2}=-\mu z_{2}\left[z_{1}^{2}+z_{2}^{2}-n W\right]+\omega z_{1}
\end{aligned}
$$

where $W \geq 0$ is a function of the states of the robot and will be formulated later. Due to the state dependent nature of the damping, the oscillator is different from the linear oscillator in Eq. (6). Also, because $W$ is not constant, it is different from the Van der Pol oscillator. If $z_{1}$ and $z_{2}$ are transformed from Cartesian to polar coordinates, $r$ and $\psi$, the oscillator takes the form ${ }^{20}$

$$
\begin{aligned}
& \dot{r}=\mu r\left(n W-r^{2}\right) \\
& \dot{\psi}=\omega
\end{aligned}
$$

The controllers presented in the next two sections are based on the nonlinear oscillator models given by Eqs. (7) and (8), respectively.

\section{ASYMPTOTIC FEEDBACK STABILIZATION STRATEGY}

\subsection{The Stabilizing Controller}

In this section we use the nonlinear oscillator in Eq. (7) to design a stabilizing controller for the mobile robot described by the kinematic model in Eq. (1). We propose the input $u_{1}$ as

$$
\begin{aligned}
& u_{1}=\mu d\left(d^{2}+\eta^{2}-n V\right)+\omega n \\
& \dot{\eta}=-\mu \eta\left(d^{2}+\eta^{2}-n V\right)+\omega d \quad \eta^{2}(0)=n V(0)
\end{aligned}
$$

where $\mu, n$, and $\omega$ are strictly positive controller parameters, and $V$ is a scalar function of the robot states defined as

$$
V=\frac{1}{2}\left(p^{2}+\Delta \theta^{2}\right)
$$

Using Eq. (3b), it can be shown that Eq. (9) represents the nonlinear oscillator,

$$
\begin{aligned}
& \dot{d}=-\mu c d-\omega \eta \\
& \dot{\eta}=-\mu c \eta+\omega d
\end{aligned} \quad c \triangleq\left(d^{2}+\eta^{2}-n V\right)
$$


The control input $u_{2}$ is proposed in terms of $u_{1}$, and the controller parameter $\alpha$, as follows,

$$
\begin{aligned}
u_{2} & =p \operatorname{sinc}(\Delta \theta) u_{1}+\alpha \Delta \theta \quad \alpha>0 \\
\operatorname{sinc}(\Delta \theta) & \triangleq \begin{cases}\frac{\sin (\Delta \theta)}{\Delta \theta} & \text { if } \Delta \theta \neq 0 \\
1 & \text { if } \Delta \theta=0\end{cases}
\end{aligned}
$$

\subsection{Asymptotic Stability}

We first observe an important property of the damping coefficient of the oscillator in Eq. (11).

Lemma: The damping coefficient of the nonlinear oscillator in Eq. (11) satisfies

$$
c(t)=d^{2}+\eta^{2}-n V \geq 0 \quad \forall t
$$

Proof: Using Eqs. (3a)-(3c), (11), and (12), the derivative of $c(t)$ can be shown to be

$$
\begin{aligned}
\dot{c}(t) & =2 d \dot{d}+2 \eta \dot{\eta}-n \dot{V} \\
& =-2 \mu\left(d^{2}+\eta^{2}\right)\left(d^{2}+\eta^{2}-n V\right)-n(p \dot{p}-\Delta \theta \dot{\theta}) \\
& =-2 \mu\left(d^{2}+\eta^{2}\right) c(t)+\alpha n \Delta \theta^{2}
\end{aligned}
$$

Now, let

$$
h(t, \tau) \triangleq \exp \left[-2 \mu \int_{\tau}^{t}\left[d^{2}(s)+\eta^{2}(s)\right] d s\right]
$$

then, we can write

$$
c(t)=h(t, 0) c(0)+\alpha n \int_{0}^{t} h(t, \tau) \Delta \theta^{2}(\tau) d \tau
$$

Since $h(t, \tau)>0, \forall t, \tau, \Delta \theta^{2}(\tau) \geq 0, \forall \tau$, and $c(0)=$ $d^{2}(0)+\eta^{2}(0)-n V(0)=d^{2}(0) \geq 0$, we can conclude $c(t) \geq 0 \forall t$.

We are now ready to establish the asymptotic stability of the mobile robot system under the control laws specified by Eqs. (9) and (12). We first show that the robot asymptotically converges to its desired posture. We then show that the desired posture is stable.

Theorem 1 (Asymptotic convergence to the desired posture): Under the control law given by Eqs. (9) and (12), the mobile robot described by Eq. (3) converges asymptotically from any initial posture to the desired posture. Specifically, $(p, d, \Delta \theta, \eta)$ will remain bounded and $(p, d, \Delta \theta, \eta) \rightarrow(0,0,0,0)$ as $t \rightarrow \infty$.

Proof: Consider a continuously differentiable function,

$$
V_{1}=\frac{1}{2}\left(p^{2}+\Delta \theta^{2}\right)+\frac{1}{2}\left(d^{2}+\eta^{2}\right)
$$

that is globally positive definite and radially unbounded. It is easy to verify that its derivative along the trajectories of the closed loop system is

$$
\dot{V}_{1}=-\alpha \Delta \theta^{2}-\mu\left(d^{2}+\eta^{2}\right) c(t)
$$

Since $c(t) \geq 0 \forall t, \dot{V}_{1} \leq 0 \forall t$. This implies that $V_{1}$ is a nonincreasing function that is bounded from below by 0 . Since $V_{1}$ is positive definite and radially unbounded, the trajectories of $(p, d, \Delta \theta, \eta)$ are bounded.

Since $V_{1}$ is nonincreasing and bounded from below by $0, V_{1}$ converges to a limit as $t \rightarrow \infty$. Since $(\dot{p}, \dot{d}, \Delta \dot{\theta}, \dot{\eta})$ are functions of $(p, d, \Delta \theta, \eta)$, as seen from Eqs. (3a), (3c), (9), (11), and (12), and $(p, d, \Delta \theta, \eta)$ are bounded, $(\dot{p}, \dot{d}, \Delta \dot{\theta}, \dot{\eta})$ are bounded. Therefore, $(p, d, \Delta \theta, \eta)$ are uniformly continuous. This implies $\dot{V}_{1}$ is uniformly continuous. Since $V_{1}$ converges to a limit and $\dot{V}_{1}$ is uniformly continuous, we can conclude from Barbalat's lemma ${ }^{21}$ that $\dot{V}_{1} \rightarrow 0$ as $t \rightarrow \infty$.

Let $L^{+}$be the positive limit set ${ }^{22}$ of $(p, d, \Delta \theta, \eta)$. Then it is invariant and satisfies the limiting condition $\dot{V}_{1}=0$. Hence,

$$
\begin{aligned}
L^{+} \subset & \left\{(p, d, \Delta \theta, \eta): \dot{V}_{1}=0\right\} \\
= & \left\{(p, d, \Delta \theta, \eta): \Delta \theta=0, d^{2}+\eta^{2}=0\right\} \\
& \cup\{(p, d, \Delta \theta, \eta): \Delta \theta=0, c(t)=0\}
\end{aligned}
$$

When $d^{2}+\eta^{2}=0, d=0$, and $\eta=0$. From the definition of $c(t)$ in Eq. (13) and $V$ in Eq. (10), this implies $p=0$ and $\Delta \theta=0$. Therefore, we have $(p, d, \Delta \theta, \eta)=(0,0,0,0)$. When $c(t)=0$, the closed loop system trajectories take the form,

$$
\begin{array}{ll}
\dot{d}=-\omega \eta & \dot{\eta}=\omega d \\
\dot{p}=0 & \dot{\Delta} \theta=-p \omega \eta
\end{array}
$$


The first two equations above can be obtained from Eq. (11), the third can be deduced from Eqs. (3a) and (9), and the fourth from Eqs. (3c) and (12). Since $\Delta \theta$ is identically zero, the fourth equation gives rise to two possibilities, namely, $p=0$ and $\eta=0$. If $p=0$, we have $V=0$ since $\Delta \theta=0$. From the definition of $c(t)$ in Eq. (13), we can then argue $d=0$ and $\eta=0$. This implies $(p, d, \Delta \theta, \eta)=(0,0,0,0)$. If $\eta$ is identically zero, $\dot{\eta}=0$ and the second equation above leads to $d=0$. Since $d=\eta=0$, we can establish from the definition of $c(t)$ and $V$ that $(p, d, \Delta \theta, \eta)=(0,0,0,0)$. From Eq. (18) we can therefore conclude that

$$
L^{+} \subset\left\{\dot{V}_{1}=0\right\} \quad \Rightarrow \quad L^{+}=\{0\}
$$

This proves that $(p, d, \Delta \theta, \eta) \rightarrow(0,0,0,0)$, or specifically $(p, d, \Delta \theta) \rightarrow(0,0,0)$ as $t \rightarrow \infty$.

Remark: The above theorem establishes asymptotic convergence of the mobile robot to its desired posture. However, it does not claim stability of the closed loop system in the four-dimensional space $(p, d, \Delta \theta, \eta)$ even though $\dot{V}_{1}$ in Eq. (18) is negative semidefinite. This is true since $\eta(0)$, which is set according to the initial posture of the robot, cannot be arbitrary. In the three-dimensional space of robot postures, we can actually claim uniform stability of the desired posture, as illustrated by the corollary below.

Corollary (Stability of the desired posture): Consider the dynamics of $(p, d, \Delta \theta)$ governed by Eqs. (3), (9), and (12). The desired posture $(p, d, \Delta \theta)=(0,0,0)$ is a uniformly stable equilibrium point.

Proof: First, $(p, d, \Delta \theta)=(0,0,0)$ is an equilibrium because if $(p(0), d(0), \Delta \theta(0))=(0,0,0)$, then $\eta(0)=0$ and $(p(t), d(t), \Delta \theta(t), \eta(t))=(0,0,0,0) \forall t \geq 0$. Next for any $\epsilon>0$, choose $\delta=2 \epsilon / \sqrt{n+2}$. Then, $\| p(0)$, $d(0), \Delta \theta(0) \| \leq \delta$ implies

$$
\begin{aligned}
V_{1}(0)= & \frac{1}{2}\left[p^{2}(0)+\Delta \theta^{2}(0)\right]+\frac{1}{2}\left[d^{2}(0)+\eta^{2}(0)\right] \\
= & \frac{1}{2}\left[p^{2}(0)+\Delta \theta^{2}(0)\right] \\
& +\frac{1}{2}\left[d^{2}(0)+\frac{n}{2}\left(p^{2}(0)+\Delta \theta^{2}(0)\right)\right] \\
\leq & \left(\frac{n+2}{4}\right) \delta^{2}
\end{aligned}
$$

and $\forall t \geq 0$,

$$
\begin{aligned}
\|p(t), d(t), \Delta \theta(t)\|^{2} \\
\quad=p^{2}(t)+d^{2}(t)+\Delta \theta^{2}(t) \\
\leq p^{2}(t)+d^{2}(t)+\eta^{2}(t)+\Delta \theta^{2}(t) \\
\quad=2 V_{1}(t) \leq 2 V_{1}(0) \leq\left[\frac{n+2}{4}\right] \delta^{2}=\epsilon^{2}
\end{aligned}
$$

The stability of the desired posture is concluded from $\|p(t), d(t), \Delta \theta(t)\| \leq \epsilon \forall t \geq 0$.

\section{ALMOST SMOOTH TIME-INVARIANT FEEDBACK CONTROL STRATEGY}

\subsection{Transformation of Variables}

In this section we use the nonlinear oscillator in Eq. (8) to design a time invariant and almost-smooth ${ }^{18}$ controller for the mobile robot. We begin with the robot model in Eq. (5) but we transform the state and control variables for the purpose of controller design. By treating the variables $x_{1}$ and $x_{2}$ in Eq. (5) as Cartesian coordinates, we transform them into polar coordinates $r$ and $\psi$ as follows,

$$
\begin{aligned}
& x_{1}=r \cos \psi \\
& x_{2}=r \sin \psi
\end{aligned} \quad r \geq 0
$$

The inverse transformation from $(r, \psi) \rightarrow\left(x_{1}, x_{2}\right)$ given by Eq. (22) is defined everywhere. The forward transformation is however undefined at the point $\left(x_{1}, x_{2}\right)=(0,0)$, where $r=0$. At all other points, the forward transformation is defined as

$$
\begin{aligned}
& r=\sqrt{x_{1}^{2}+x_{2}^{2}} \\
& \psi=\arctan \frac{x_{2}}{x_{1}} \quad r \neq 0
\end{aligned}
$$

By differentiating Eq. (22) and using Eq. (5), we get

$$
\begin{aligned}
& v_{1}=\dot{x}_{1}=\dot{r} \cos \psi-r \sin \psi \dot{\psi} \\
& v_{2}=\dot{x}_{2}=\dot{r} \sin \psi+r \cos \psi \dot{\psi}
\end{aligned}
$$


Substitution of Eqs. (22) and (24) into Eq. (5c) results in

$$
\begin{array}{ll}
\dot{x}_{3}=\eta_{1}(r, \psi) \dot{r}+\eta_{2}(r, \psi) \dot{\psi} \quad & \eta_{1} \triangleq r \sin \psi \cos \psi \\
& \eta_{2} \triangleq-r^{2} \sin ^{2} \psi
\end{array}
$$

Now, we transform $x_{3}$ in Eq. (5) into a new variable, which we denote by $\beta$, using the relation,

$$
\begin{aligned}
\beta & =x_{3}-\int_{0}^{r} \eta_{1} d r=\left(x_{3}-\frac{r^{2}}{2} \sin \psi \cos \psi\right) \\
& =\left(x_{3}-\frac{1}{2} x_{1} x_{2}\right)
\end{aligned}
$$

In the transformed coordinates, the kinematics of the robot takes the form,

$$
\begin{aligned}
& \dot{r}=w_{1} \\
& \dot{\psi}=w_{2} \\
& \dot{\beta}=-\frac{r^{2}}{2} w_{2}
\end{aligned}
$$

where Eq. (27c) was obtained by differentiating Eq. (26) and substituting Eq. (25). The convergence of the mobile robot to its desired posture now refers to the convergence of $r$ and $\beta$ to zero. Indeed, from Eqs. (23) and (26), $(r, \beta)=(0,0)$ implies $\left(x_{1}, x_{2}, x_{3}\right)$ $=(0,0,0)$.

\subsection{The Feedback Control Strategy}

The feedback control strategy for the kinematic model of Eq. (27) is proposed as

$$
\begin{aligned}
& w_{1}=\alpha r\left(\sigma^{2} \beta^{2}-r^{2}\right) \\
& w_{2}=\gamma \beta
\end{aligned}
$$

where, it is assumed that

$$
r(0) \neq 0
$$

and where $\alpha, \gamma$, and $\sigma$ are positive controller parameters, chosen according to the relation,

$$
\gamma=2 \alpha\left(1+\sigma^{2}\right)
$$

The actual controls for the robot, $v_{1}$ and $v_{2}$ defined in Eq. (5), are related to the control variables $w_{1}$ and $w_{2}$ in Eq. (27) via Eq. (24). The control inputs for the asymptotic convergence of the states of the robot are therefore proposed as

$$
v_{1}= \begin{cases}r\left[\alpha\left(\sigma^{2} \beta^{2}-r^{2}\right) \cos \psi-\gamma \beta \sin \psi\right] & \text { if } r>0 \\ 0 & \text { if } r=0\end{cases}
$$

$$
v_{2}= \begin{cases}r\left[\alpha\left(\sigma^{2} \beta^{2}-r^{2}\right) \sin \psi+\gamma \beta \cos \psi\right] & \text { if } r>0 \\ 0 & \text { if } r=0\end{cases}
$$

The controller proposed in Eq. (32) cannot stabilize the states of the system to the origin from points where $r(0)=0$, or $x_{1}(0)=x_{2}(0)=0$. Nevertheless, system states arbitrarily close to, but not exactly on, the manifold of equilibria $r=0$ can be asymptotically converged to the equilibrium state-this is explained in the next remark, and proved in section 5.3

Remark: The assumption in Eq. (30) is reasonable since $r(0)$ cannot be exactly equal to zero. For the system defined by Eqs. (27a) and (28), it is apparent that $r=0$ is an unstable equilibrium manifold for $\beta \neq 0$. Therefore the assumption in Eq. (30) ensures that the system trajectories do not remain stuck at this unstable equilibrium manifold.

Remark: The controller in Eq. (32) is (i) defined everywhere on $R^{3}$, (ii) smooth on the open (and dense) subset $R^{3} \backslash\{r=0\}$ of $R^{3}$, and (iii) continuous at $r=0$. Therefore they are almost-smooth ${ }^{18}$ functions of the states of the mobile robot. Other controllers ${ }^{12,13}$ using nonsmooth transformation do not satisfy the third condition. The controller in Eq. (32) is also time-invariant as seen from Eqs. (23) and (26).

\subsection{Stability and Convergence}

In this section we establish that the mobile robot converges to its desired posture $\left(x_{1}, x_{2}, x_{3}\right)=(0,0,0)$ from every posture satisfying Eq. (30) for the control inputs defined by Eq. (32). To this end we establish $(r, \beta) \rightarrow(0,0)$ for the control inputs defined by Eqs. (28) and (29). We also show in this section that the desired posture $\left(x_{1}, x_{2}, x_{3}\right)=(0,0,0)$ is stable. 
Theorem 2 (Asymptotic convergence to the desired posture): Under the control law given by Eqs. (28) and (29), the mobile robot described by Eq. (27) converges asymptotically from any initial posture satisfying $r(0) \neq$ 0 , to the desired posture. Specifically $(r, \beta) \rightarrow(0,0)$ as $t \rightarrow \infty$.

Proof: Substituting Eqs. (28) and (29) into Eq. (27), the dynamics of $(r, \beta)$ is seen to be

$$
\begin{aligned}
& \dot{r}=\alpha r\left(\sigma^{2} \beta^{2}-r^{2}\right) \\
& \dot{\beta}=-\left(\frac{\gamma}{2}\right) r^{2} \beta
\end{aligned}
$$

It is apparent from Eq. (33a) that $r=0$ represents a set of unstable equilibria for any fixed $\beta \neq 0$. Therefore, unless $(r, \beta)=(0,0)$, the robot cannot practically stay at $r=0$. Let us therefore assume that $r(0) \neq 0$ and show that $(r, \beta) \rightarrow(0,0)$ as $t \rightarrow \infty$.

Consider the state space $X$ in $(r, \beta)$ to be $X=$ $X_{0} \cup X_{+}, X_{0}=(0,0), X_{+}=\{(r, \beta): r>0\}$. In this space, define the Lyapunov function $\hat{V}$ in the following way. Pick $c \geq 0$ and let $(c, 0)$ be a point on the $r$ axis in the $(r, \sigma \beta)$ half plane, as shown in Figure 2a. Draw a circle that is symmetric about the $r$ axis and passes through $(0,0)$ and $(c, 0)$. Let $\hat{V}$ be defined such that its level set $\hat{V}=c$ is the circle, i.e.,

$$
\left(\frac{\hat{V}}{2}\right)^{2}=\left(r-\frac{\hat{V}}{2}\right)^{2}+\sigma^{2} \beta^{2}
$$

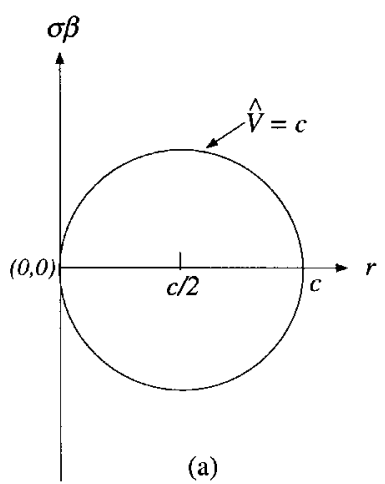

(a)

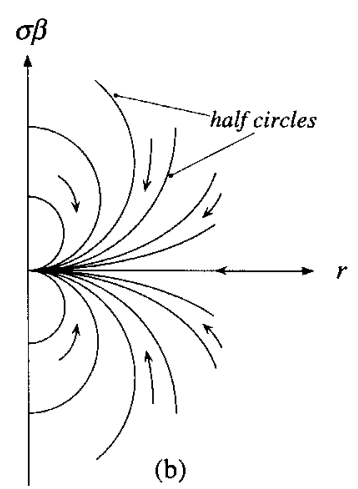

Figure 2. (a) Circle in the $r-\sigma \beta$ plane defining the Lyapunov function $\hat{V}$, (b) Phase portraits in the $r-\sigma \beta$ plane showing the convergence of $r$ and $\beta$ to $(0,0)$.
Clearly, $\hat{V} \geq 0$ in $X$ and $\hat{V}=0$ implies $(r, \beta)=(0,0)$. Furthermore, if $\|(r, \beta)\| \rightarrow \infty, \hat{V} \rightarrow \infty$. Therefore, $\hat{V}$ is positive definite and radically unbounded. Note, however, when $r=0, \hat{V}$ is only defined for $\beta=0$ since Eq. (34) becomes $(\hat{V} / 2)^{2}=(\hat{V} / 2)^{2}+\sigma^{2} \beta^{2}$. This is not a problem since $\hat{V}$ is defined in the set $X$.

Before we calculate the derivative of $\hat{V}$ we rewrite Eq. (34) in the following two forms,

$$
\begin{gathered}
r^{2}-r \hat{V}=-\sigma^{2} \beta^{2} \\
2 r^{2}-r \hat{V}=r^{2}-\sigma^{2} \beta^{2}
\end{gathered}
$$

Differentiating Eq. (35a) with respect to time, substituting Eqs. (33) and (35b), and rearranging terms yields

$$
\begin{aligned}
r \dot{\hat{V}} & =2 r \dot{r}-\dot{r} \hat{V}+2 \sigma^{2} \beta \dot{\beta} \\
& =\alpha\left(2 r^{2}-r \hat{V}\right)\left(\sigma^{2} \beta^{2}-r^{2}\right)-\gamma \sigma^{2} r^{2} \beta^{2} \\
& =-\alpha\left(\sigma^{2} \beta^{2}-r^{2}\right)^{2}-\gamma \sigma^{2} r^{2} \beta^{2}
\end{aligned}
$$

Hence $r \dot{\hat{V}}$ is negative definite in $X$ and is equal to zero at $(r, \beta)=(0,0)$. Since $\hat{V}$ is positive definite and radially unbounded in $X$, and $\dot{\hat{V}}$ is negative definite in $X$, we conclude that for all $(r(0)), \beta(0))$ $\in X, \hat{V} \rightarrow 0$ as $t \rightarrow \infty$. This implies that $(r, \beta) \rightarrow(0,0)$ as $t \rightarrow \infty$.

Discussion: It is interesting to examine the phase portraits in $X$. This will give an alternative graphical explanation of the convergence of $(r, \beta)$ to $(0,0)$. From Eq. (33), we get

$$
\frac{d r}{d \beta}=-\frac{2 \alpha\left(\sigma^{2} \beta^{2}-r^{2}\right)}{\gamma r \beta}
$$

Let us choose $\alpha=\gamma / 4$ for simplicity. Then, manipulation of the above equation leads to

$$
r^{2}+\left(\sigma^{2} \beta^{2}-\kappa \beta\right)=0
$$

where $\kappa$ is a constant of integration. For each value of $\kappa$, $E q$. (37) gives one orbit of the $(r, \beta)$ dynamics in the phase plane. These orbits are half circles in $X$ and are shown in Figure $2 b$. Clearly, all the trajectories converge to $(r, \beta)=(0,0)$ as $t \rightarrow \infty$. 
Remark: The above theorem establishes asymptotic convergence of the mobile robot to its desired posture $(r, \beta)=(0,0)$ or $\left(x_{1}, x_{2}, x_{3}\right)=(0,0,0)$. It does not claim stability of the desired posture even though $\hat{V}$ in Eq. (36) is negative definite because $\hat{V}$ was defined in the subset $X, X=X_{0} \cup X_{+}, X_{0}=$ $(0,0), X_{+}=\{(r, \beta): r>0\}$, of the posture space. In the complete space of robot postures, we claim stability of the desired posture with the help of the corollary below.

Corollary (Stability of the desired posture): Consider the dynamics of the mobile robot described by Eqs. (5) and (32). The robot posture $\left(x_{1}, x_{2}, x_{3}\right)=(0,0,0)$ is a uniformly stable equilibrium point.

Proof: Define the Lyapunov function candidate,

$$
V^{\prime}=x_{1}^{2}+x_{2}^{2}+\left(x_{3}-\frac{x_{1} x_{2}}{2}\right)^{2}
$$

Clearly $V^{\prime} \geq 0$ and $V^{\prime}=0$ implies $\left(x_{1}, x_{2}, x_{3}\right)=$ $(0,0,0)$. Therefore $V^{\prime}$ is globally positive definite. Using Eqs. (5c), (22), (26), (31), and (32), the derivative of $V^{\prime}$ can be computed as

$$
\begin{aligned}
\dot{V}^{\prime}= & x_{2} \dot{x}_{1}+x_{2} \dot{x}_{2} \\
& +\left(x_{3}-\frac{x_{1} x_{2}}{2}\right)\left(\dot{x}_{3}-\frac{x_{1}}{2} \dot{x}_{2}-\frac{x_{2}}{2} \dot{x}_{1}\right) \\
= & \left(x_{1} v_{1}+x_{2} v_{2}\right)+\frac{\beta}{2}\left(x_{2} v_{1}-x_{1} v_{2}\right) \\
= & \alpha r^{2}\left(\sigma^{2} \beta^{2}-r^{2}\right)-\frac{\gamma}{2} r^{2} \beta^{2} \\
= & -\alpha r^{2}\left(r^{2}+\beta^{2}\right) \\
\leq & 0
\end{aligned}
$$

The uniform stability of $\left(x_{1}, x_{2}, x_{3}\right)=(0,0,0)$ follows from the negative semidefiniteness of $\dot{V}^{\prime}$.

Remark: The two control strategies presented in this article use the same nonlinear oscillator. The oscillator frequency of the first controller, denoted by $\omega$ in Eqs. (7) and (9) is a constant. For the controller presented in this section, the frequency, denoted by $\dot{\psi}$, is a variable that converges to zero. This is evident from Eqs. (8) and (29) and the fact that $\beta$ converges to zero. As a result, the phase of the oscillator, $\psi$, converges to some value which is not important.

\section{SIMULATION RESULTS}

Simulation results are presented here to demonstrate the capability of the two controllers to converge the mobile robot to different desired postures. For the controller in section 4, the initial and final coordinates of the robot are given in Table I, where the units are meters and degrees. Case A represents the parallel parking problem. The trajectory of the robot for this case is shown in Figure 3A. In cases $\mathrm{B}-\mathrm{D}$, the robot makes a 360 , a 135 , and a $-180^{\circ}$ turn, respectively, between its initial and final configurations. The trajectories of the robot for these cases are shown in Figure 3B-D, respectively.

The initial and final configurations of the mobile robot are interchanged for the almost-smooth time-invariant controller in section 5. The simulation results are shown in Figure $4 \mathrm{~A}-\mathrm{D}$, respectively. Of particular interest is Case $\mathrm{A}$, where $r(0)=0$. This violates the assumption in Eq. (30). The problem is remedied by assuming the initial orientation to be $0.01^{\circ}$ instead of exactly zero. A comparison of Figures 3 and 4 indicate that the two controllers generate qualitatively different trajectories.

\section{CONCLUSION}

Undamped linear oscillators have been widely used in the point-to-point control of mobile robots and nonholonomic systems but they offer limited flexi-

\begin{tabular}{|c|c|c|c|c|c|c|}
\hline & $\left(x_{i}\right.$ & $y_{i}$ & $\left.\theta_{i}\right)$ & $\left(x_{d}\right.$ & $y_{d}$ & $\left.\theta_{d}\right)$ \\
\hline Case A & (0 & 0 & 0) & $(0$ & 1 & 0) \\
\hline Case B & (0 & 0 & 0) & ( 1 & 1 & 360) \\
\hline Case C & (0 & 0 & 0) & $(-5$ & 3 & 135) \\
\hline Case D & (0 & 0 & 0) & ( 1 & 0 & $-180)$ \\
\hline
\end{tabular}
bility due to their fixed structure. In contrast, a large number of qualitatively different control strategies can be designed using nonlinear oscillators, since their stiffness and damping can be func-

Table I. Initial and final configurations of the mobile robot. 


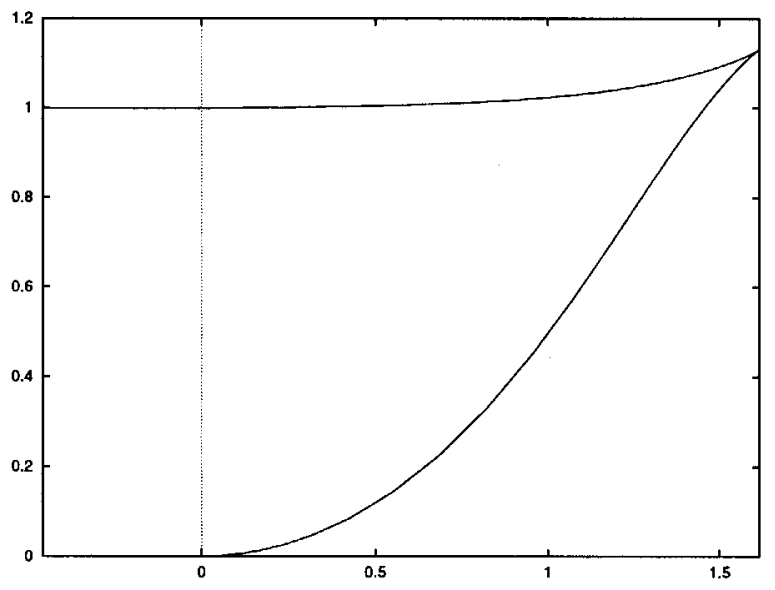

Case (A)

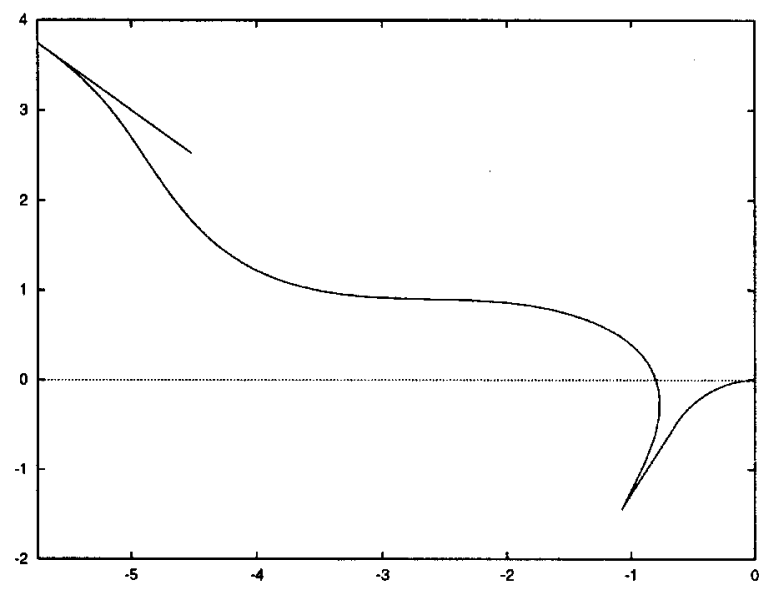

Case (C)

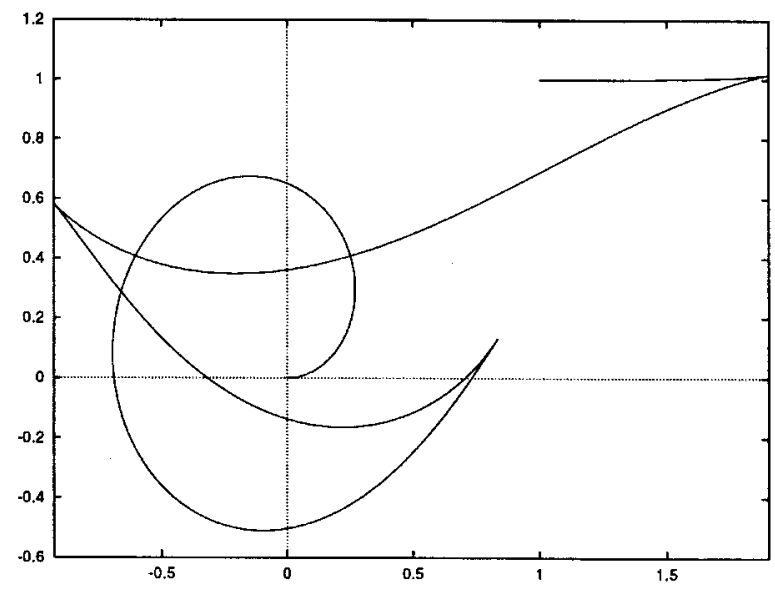

Case (B)

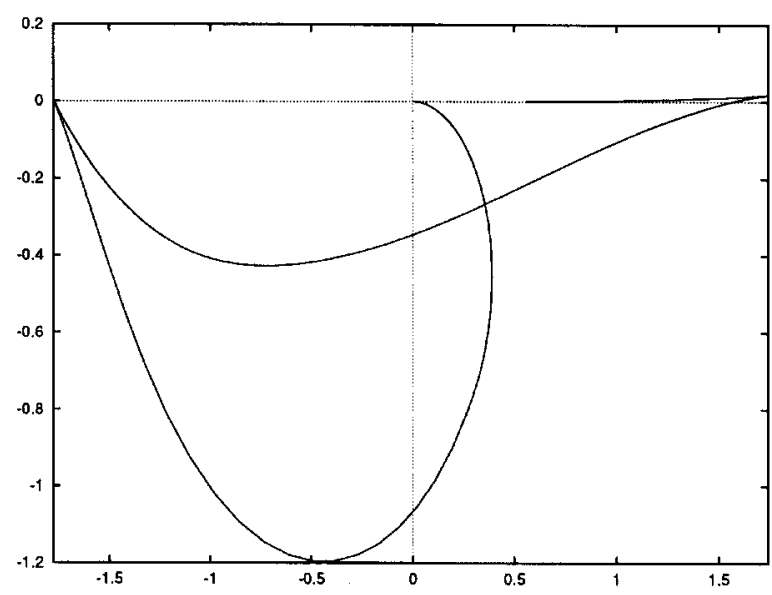

Case (D)

Figure 3. Mobile robot trajectories for the stabilizing dynamic controller in section 4 .

tions of the robot states. In this article we use two variants of a nonlinear oscillator to design two fundamentally different control strategies for a twowheeled mobile robot. The first controller is smooth and dynamic and guarantees asymptotic stability for any desired posture. The second strategy provides stability and asymptotically converges the closed loop system trajectories from practically any posture to the desired posture using almost-smooth and time-invariant controls. It uses the chained- form ${ }^{19}$ kinematic model and provides a means of generalization to other nonholonomic systems. For both controls strategies, we mathematically establish stability and convergence of the closed loop system trajectories to the desired posture. We also provide numerical simulations to demonstrate the efficacy of the control strategies. An interesting feature of both strategies is their ability to handle situations where the robot has to turn more than $360^{\circ}$ between initial and final postures. Such capa- 


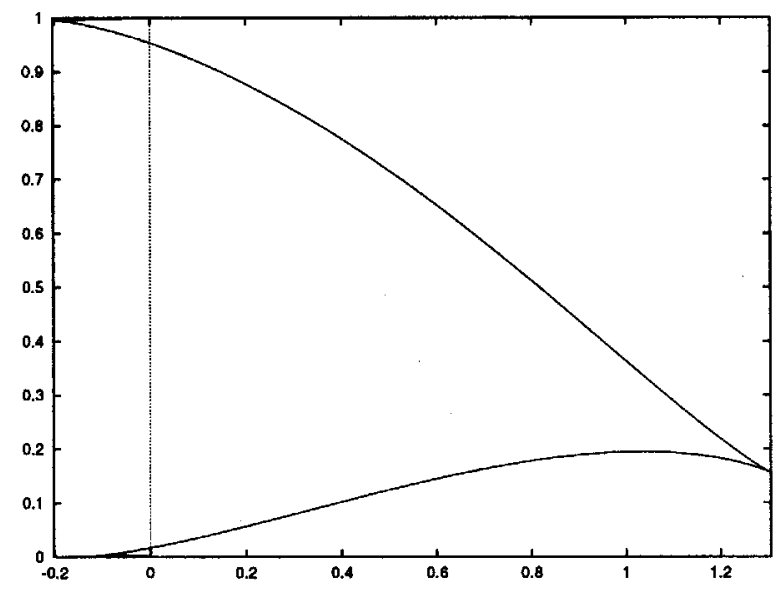

Case (A)

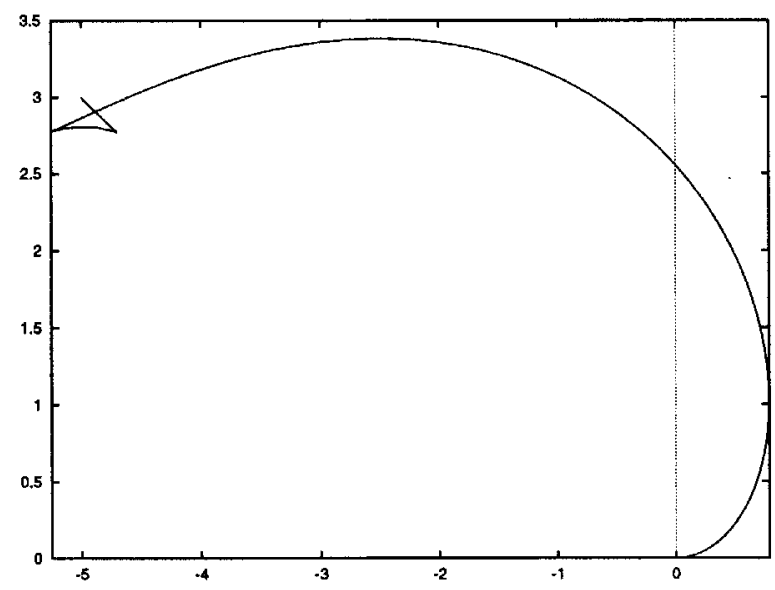

Case (C)

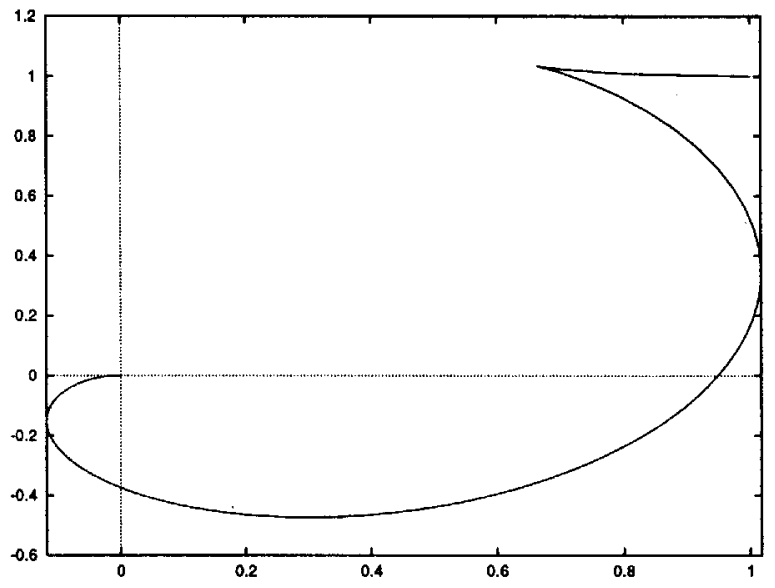

Case (B)

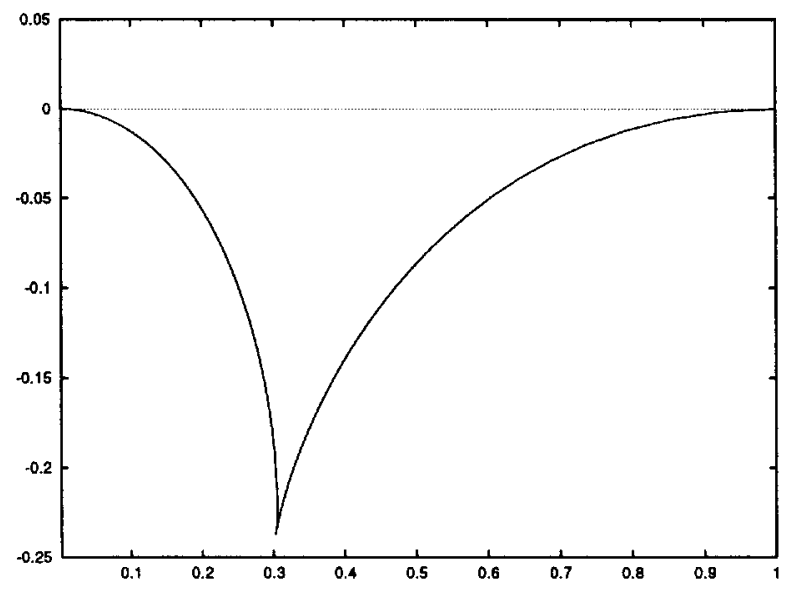

Case (D)

Figure 4. Mobile robot trajectories for the almost-smooth time-invariant controller in section 5 .

bility will be useful for tethered mobile robots in factory automation. In the past nonlinear oscillators have not been used for the control of mobile robots or nonholonomic systems and the results here suggest that additional investigations should be made for their applicability to higher order systems.

The first and second authors gratefully acknowledge the support provided by the National Science Foundation, NSF Grant Nos. CMS-9410157 and ECS9410646, respectively.

\section{REFERENCES}

1. R.W. Brockett, "Asymptotic stability and feedback stabilization," Differential geometric control theory, R.W. Brockett, R.S. Millman, and H.J. Sussman (Editors), Birkhäuser, Basel, 1983, pp. 181-208.

2. I. Kolmanovsky and N.H. McClamroch, Developments in nonholonomic control problems, IEEE Contr Syst 15 (1995), 20-36.

3. C. Samson, "Velocity and torque feedback control of a nonholonomic cart," Int Workshop in Adaptive and Nonlinear Control: Issues in Robotics, Proc Advanced 
Robot Control, C. Canudas de Wit (Editor), SpringerVerlag, Berlin/New York, Vol. 162, 1990, pp. 125-151.

4. J.M. Coron, Global asymptotic stabilization for controllable systems without drift, Math Contr Signals Syst 5 (1992), 295-312.

5. J.B. Pomet, Explicit design of time-varying stabilizing control laws for a class of controllable systems without drift, System and Control Letters 18 (1992), 147-158.

6. R. Mukherjee, D. Chen, and G. Song, Asymptotic feedback stabilization of a nonholonomic mobile robot using a nonlinear oscillator, IEEE Conf on Decision and Control, 1996, pp. 1422-1427.

7. L. Gurvits and Z.X. Li, Smooth time-periodic feedback solutions for nonholonomic motion planning, Progress in nonholonomic motion planning, Z.X. Li and J. Canny (Editors), Kluwer Academic, Dordrecht/Norwell, MA, 1992, pp. 53-108.

8. R.T. McCloskey and R.M. Murray, Convergence rates for nonholonomic systems in power form, American Control Conf, San Francisco, CA, 1993.

9. H.J. Sussmann, Subanalytic sets and feedback control, J Differential Equations 31 (1979), 31-52.

10. A.M. Bloch, N.H. McClamroch, and M. Reyhanoglu, Controllability and stability properties of a nonholonomic control system, IEEE Conf on Decision and Control, 1990, pp. 1312-1314.

11. C. Canudas de Wit and O.J. Sordalen, Exponential stabilization of mobile robots with nonholonomic constraints, IEEE Trans Automat Contr 37 (1992), 1791-1797.
12. M. Aicardi, G. Casalino, A. Balestrino, and A. Bicchi, Closed-loop smooth steering of unicycle-like vehicles, IEEE Conf on Decision and Control, 1994, pp. 24552458.

13. A. Astolfi, On the stabilization of nonholonomic systems, IEEE Conf on Decision and Control, 1994, pp. 3481-3486.

14. J. Guldner and V.I. Utkin, Stabilization of nonholonomic mobile robots using Lyapunov functions for navigation and sliding mode control, IEEE Conf on Decision and Control, 1994, pp. 2967-2972.

15. A.M. Bloch and S. Drakunov, Stabilization of a nonholonomic system via sliding modes, IEEE Conf on Decision and Control, 1994, pp. 2961-2963.

16. A.M. Bloch, M. Reyhanoglu, and N.H. McClamroch, Control and stabilization of nonholonomic dynamical systems, IEEE Trans Automat Contr 37 (1992), 17461757.

17. O.J. Sordalen and O. Egeland, Exponential stabilization of nonholonomic chained systems, IEEE Trans Automat Contr 40 (1995), 35-49.

18. A. Isidori, Nonlinear control systems, 3rd ed., Springer-Verlag, New York, 1995.

19. R.M. Murray and S.S. Sastry, Nonholonomic motion planning: Steering using sinusoids, IEEE Trans Automat Contr 38 (1991), 700-713.

20. M. Vidyasagar, Nonlinear systems analysis, PrenticeHall, Englewood Cliffs, NJ, 1993.

21. J-J.E. Slotine and W. Li, Applied nonlinear control, Prentice-Hall, Englewood Cliffs, NJ, 1991.

22. H. Khalil, Nonlinear systems, 2nd ed., Prentice-Hall, Englewood Cliffs, NJ, 1996. 